\title{
Laparoscopic urinary bladder diverticulectomy combined with photoselective vaporisation of the prostate
}

\author{
Milan Hora ${ }^{1,2}$, Viktor Eret ${ }^{1,2}$, Petr Stránský ${ }^{1}$, Ivan Trávníček ${ }^{1,2}$, Olga Dolejšová ${ }^{1,2}$, Zdeněk Chudáček ${ }^{3}$, Fredrik Petersson ${ }^{4}$, \\ Ondřej Hes ${ }^{2,5}$, Piotr Chłosta ${ }^{6}$ \\ ${ }^{1}$ Department of Urology, Faculty Hospital in Pilsen, Czech Republic \\ ${ }^{2}$ Faculty of Medicine in Pilsen, Charles University in Prague, Czech Republic \\ ${ }^{3}$ Department of Radiology, Faculty Hospital, Pilsen, Czech Republic \\ ${ }^{4}$ Department of Pathology, National University Health System, Singapore \\ ${ }^{5}$ Department of Pathology, Faculty Hospital in Pilsen, Czech Republic \\ ${ }^{6}$ Department of Urology, Jagiellonian University Medical College, Krakow, Poland
}

Videosurgery Miniinv 2015; 10 (1): 62-67 DOI: $10.5114 /$ wiitm.2015.49671

\begin{abstract}
Introduction: Pseudodiverticulum of the urinary bladder is mostly a complication of subvesical obstruction (SO). The gold standard of treatment was open diverticulectomy with adenectomy. A more contemporary resolution is endoscopic, in two steps: the first transurethral resection of the prostate (TURP), the second laparoscopic diverticulectomy (LD). Aim: To present a one-session procedure - photoselective vaporisation of the prostate (PVP) with LD.

Material and methods: From 1/2011 to 6/2014, 14 LDs were performed: 1 LD only, 1 with laparoscopic radical prostatectomy, 12 combined with treatment of benign prostatic hyperplasia (BPH), 4 cases of TURP and LD in the second period. In 8 cases, PVP and LD in one session were combined. These 8 cases are presented. 3D CT cystography was used as a gold standard for assessment of diverticulum.

Results: The mean age was $66.5 \pm 5.5$ (57.3-75.1) years, the mean size of the diverticulum $61.8 \pm 22.1$ (26-90) mm. The procedure starts in the lithotomy position. It includes PVP and stenting of the ureter(s). Changing of position and laparoscopy follows: four ports, transperitoneal extravesical approach. Photoselective vaporisation of the prostate was performed using the Green Light Laser HPS (1x) or XPS with cooled fibre MoXy (7x). The mean delivered energy in PVP was $205.1 \pm 106.4(120-458) \mathrm{kJ}$. The mean time of operation was $165.0 \pm 48.5$ (90-255) min. No postoperative complications were observed. One patient underwent TUR incision after 1 year for sclerosis of the bladder neck. Conclusions: Pseudodiverticulum of the urinary bladder (with or without SO) is a relatively rare disease. One session of PVP (Green Light Laser XPS, MoXy fibre) and laparoscopic (transperitoneal extravesical) diverticulectomy is the preferred method for treatment of subvesical obstruction due to BPH and bladder diverticulum at our institution.
\end{abstract}

Key words: laparoscopic diverticulectomy, photoselective vaporisation of the prostate, benign prostatic hyperplasia.

\section{Introduction}

Urinary bladder diverticula (UBD) may be congenital or acquired. A congenital diverticulum involves all layers of the bladder wall, whereas acquired di- verticula form by the herniation of mucosa through the muscle layer of the bladder (= pseudodiverticula). Urinary bladder diverticula are mostly acquired and secondary to bladder outlet obstructions, mainly as

Address for correspondence

Prof. Milan Hora MD, PhD, Department of Urology, Faculty Hospital in Pilsen, Czech Republic and Faculty of Medicine in Pilsen,

Charles University in Prague, 13 E. Benese St, 30599 Pilsen, Czech Republic, phone: +42 0377402225, +42 0602950086,

fax: +42 0377402171, e-mail: horam@fnplzen.cz 
a result of benign prostatic hyperplasia $(\mathrm{BPH})$. Large diverticula may lead to dysuria, residual urine, and urinary tract infection (UTI). Urinary tract infection in this setting may be recurrent. Treatment of acquired UBD should consist of diverticulectomy and relief of the bladder outlet obstruction [1]. Formerly, the standard choice of treatment was open surgery - diverticulectomy combined with open prostatectomy. The first cases of laparoscopic diverticulectomy (LD) are dated to 1992 [2, 3]. Subsequently, the feasibility of LD has been validated [4-8]. Laparoscopic diverticulectomy can also be performed using a robotic system [1, 9-11]. As mentioned, the vast majority of cases of acquired UBD are combined with, and etiologically related to, subvesical obstruction, mainly $\mathrm{BPH}$, which should be alleviated. Diverticulectomy may be combined with radical prostatectomy [11]. However, this should not be performed in paediatric cases in which diverticulectomy is also indicated [12]. Questions/issues for clinical practice in $\mathrm{BPH}$ with UBD are: (1) Open or minimally invasive approach to UBD? (2) Laparoscopy or robotic surgery? (3) What type of treatment of BPH? Transurethral re- section of the prostate (TURP) or photoselective vaporisation of prostate (PVP) or another technique? (4) One- or two-step procedure?

\section{Aim}

Herein, we present our experience based on a small case series with this field of operative urology and with our preferred combination of methods, namely a two-step procedure in one session: PVP immediately followed by laparoscopic transperitoneal extravesical diverticulectomy. Due to the rarity of this diagnosis, it is difficult to perform a randomised clinical trial.

\section{Material and methods}

From 1/2011 to 6/2014, 14 LDs were performed at the institution of the main author: 1 LD only, 1 with laparoscopic radical prostatectomy and $12 \mathrm{com}$ bined with treatment of BPH. Four cases were performed in 2 sessions: first TURP followed by LD in a second session. These procedures were indicated before the availability of a PVP machine. In 8 cases,

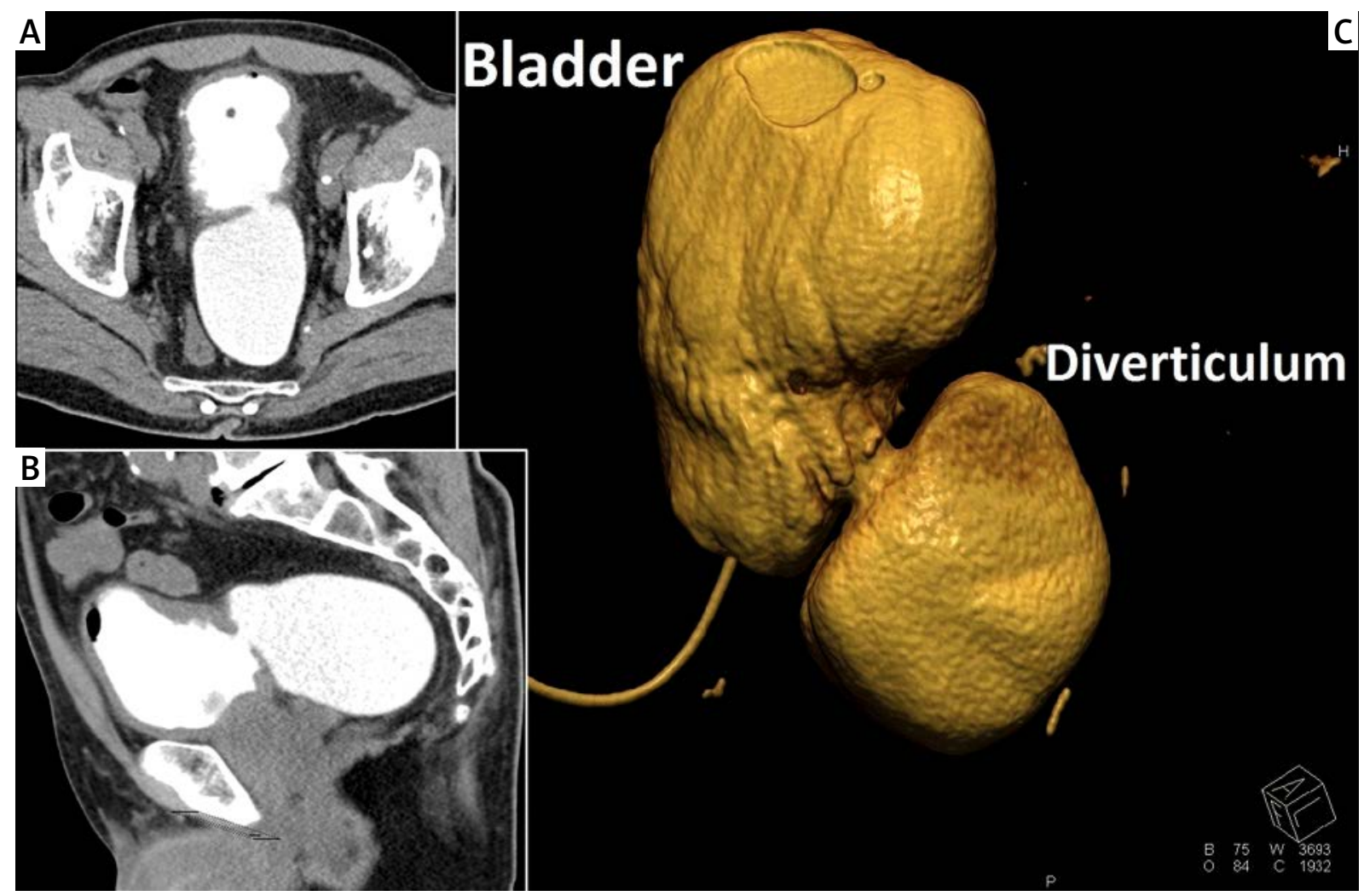

Photo 1. Assessment of bladder diverticulum on CT. A - Axial section, B - sagittal section, C - 3D reconstruction (of another case than in A and B) 


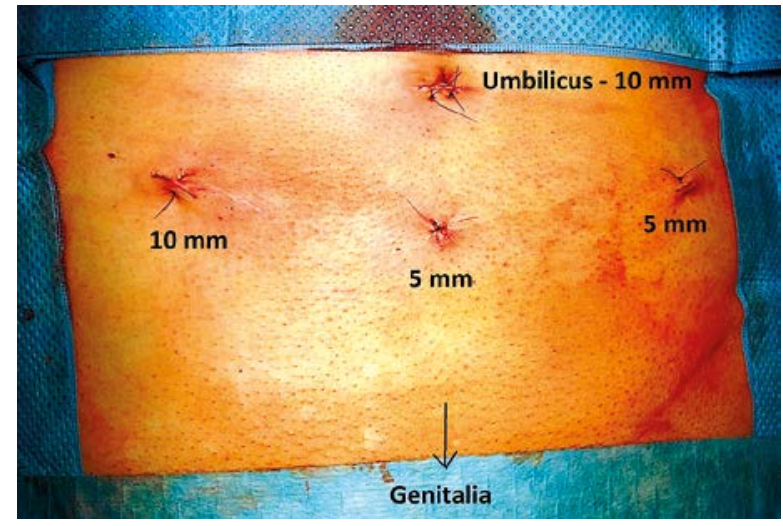

Photo 2. Abdominal wall at the end of surgery with placement of ports. The $10 \mathrm{~mm}$ port on the right site is used for introduction of needles and extraction of specimen. No drain was used in this case

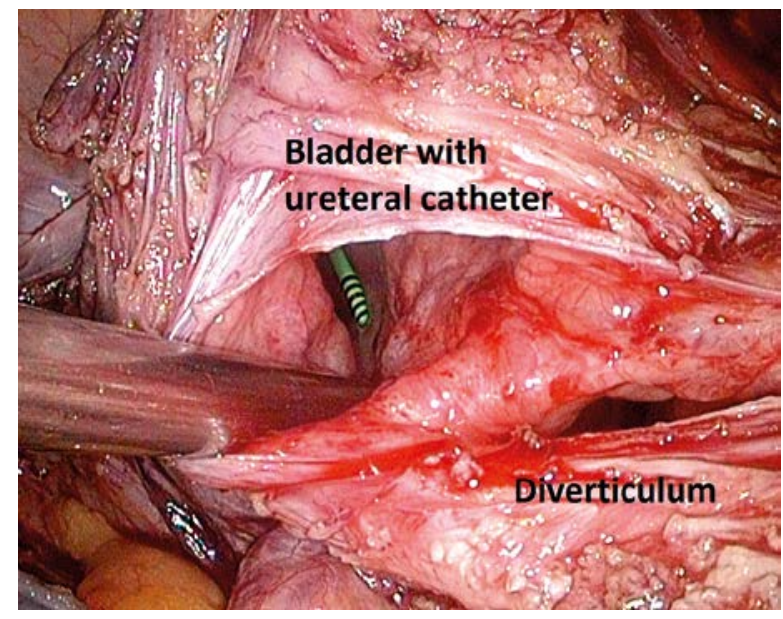

Photo 4. Laparoscopic view of opened urinary bladder (inside the ureteral catheter is visible). The diverticulum (located on the right side of the bottom of the bladder) is partially divided at the broad-based neck of the diverticulum

PVP and LD were combined in one session. These 8 cases are presented. 3D CT cystography was used as the gold standard for assessment of the diverticulum (Photo 1).

Operative technique of LD with PVP: the procedure starts in the lithotomy position. It includes uretrocystoscopy, PVP and stenting of one or both ureters. The urinary bladder catheter and ureteral catheter are wrapped in a sterile way. The position of the patient is then changed to the Trendelenburg position. The operative field is prepared again and

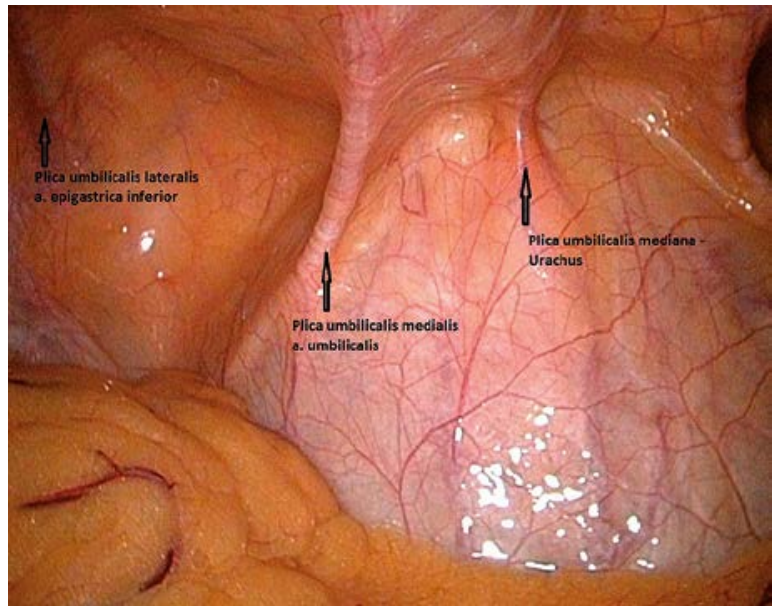

Photo 3. Laparoscopic view of anterior abdominal wall and filled urinary bladder in a slim man. Bowels had to be retracted to obtain access to the bottom of the urinary bladder

laparoscopy follows. The approach is transperitoneal extravesical. Capnoperitoneum is created with a Veress needle with pressure of $12 \mathrm{~mm} \mathrm{Hg}$. Four ports are used, $2 \times 10 \mathrm{~mm}, 2 \times 5 \mathrm{~mm}$ (Photos 2, 3). The bladder is filled with approximately $300 \mathrm{ml}$ of sterile physiological solution. The peritoneum is opened with a harmonic scalpel at the lower part of the bladder in the area of the diverticulum. The diverticulum is liberated and is divided from the bladder at the level of neck (Photo 4). Close attention should be paid to avoiding any trauma of the ureter, which is frequently located close to the diverticulum. Stenting of the ureter helps to avoid injury of the ureter. The defect in the bladder (at the site of the division of the neck of the diverticulum) is closed with running two-layer suture with polyglactin (requiring knotting) or recently with absorbable barbed suture V-Loc(TM) 90 (a mixture of glycolide, dioxanone and trimethylene). To test the watertightness of the bladder, we use $200 \mathrm{ml}$ of sterile physiological solution. The peritoneal defect is closed with another V-Loc(TM) 90 thread. The surgical specimen (diverticulum) is removed through a $10 \mathrm{~mm}$ port (which can be dilated if needed). The surgical specimen is sent for histopathological examination. A drain to the Douglas space can be inserted. Defects of the abdominal wall are closed. The ureteral catheter(s) is removed on the $1^{\text {st }}$ postoperative day, the bladder catheter on the $6^{\text {th }}$ postoperative day, mostly without previous cystography. Following micturition, the patient is discharged from hospital. 


\section{Results}

The mean age was $66.5 \pm 5.5(57.3-75.1)$ years, the mean body mass index (BMI) $27.6 \pm 4.1(23-35)$ $\mathrm{kg} / \mathrm{m}^{2}$. The mean size of the prostate was $44.5 \pm 14.7$ (30-80) $\mathrm{ml}$ and mean prostate-specific antigen (PSA) value $2.9 \pm 1.8(0.5-6.5) \mathrm{ng} / \mathrm{ml}$ (in the patient with PSA 6.5 the free/total PSA ratio was $33 \%$ ). The mean maximal size of the diverticulum on computed tomography cystography was $61.8 \pm 22.1(26-90) \mathrm{mm}$. Photoselective vaporisation of prostate was performed with Green Light Laser HPS 120 W (1x, first case) or XPS $180 \mathrm{~W}$ with cooled fibre MoXy (7x). The mean delivered energy in PVP was $205.1 \pm 106.4$ (120-458) kJ. The mean duration of surgery was $165.0 \pm 48.5$ (90-255) min. The longest time (255 min) was in an obese man with BMI $35 \mathrm{~kg} / \mathrm{m}^{2}$. All surgical procedures were performed by one surgeon. There were no peri- or postoperative complications. One patient underwent TUR incision 1 year after the procedure for sclerosis of the bladder neck - Dindo-Clavien IIIb [13]. The histological examination of all diverticula was benign in all cases.

\section{Discussion}

We have attempted to answer the four questions about the surgical treatment of UBD with subvesical obstruction due to BPH expressed in the introduction: (1) Open or minimally invasive approach? (2) Laparoscopy or robotic surgery? (3) What type of treatment of the underlying $\mathrm{BPH}-\mathrm{TURP}$, PVP or another technique? (4) A one- or two-step procedure? Firstly, however, we would like to make one comment regarding the preoperative diagnostic process. A bladder examination is a common scenario for patients with $\mathrm{BPH}$. We only plan the operation after a CT cystography including a 3D reconstruction has been performed [14]. We consider plain X-ray cystography to be insufficient. Prior to or at the start of the surgical procedure, a meticulous endoscopy is performed to exclude any bladder co-morbidities, e.g. urothelial tumour. However, LD of UBD harbouring urothelial carcinoma has been reported [15].

Regarding the technique of the diverticulectomy: Urologists are familiar with surgery in the small pelvis with a transperitoneal or extraperitoneal [8] approach to the urinary bladder mainly in the setting of laparoscopic radical prostatectomy and/or cystectomy $[16,17]$. Numerous alternative techniques of diverticulectomy have been described [14]: open (intravesical and extravesical), endoscopic (fulguration or diverticular neck resection), and laparoscopic/robotic. Laparoscopic or robotic diverticulectomy is the preferred method due to excellent efficiency and minimal invasiveness and morbidity. Laparoscopy can be extraperitoneal $[6,8]$, intraperitoneal (= transperitoneal) [2, 3] or suprapubic transvesical [18-20]. Pure laparoscopy can be replaced by robotic-assisted bladder diverticulectomy $[1,9,11,12,14$, 18, 19, 21-23]. Attempts to introduce laparoendoscopic single site surgery (LESS) have also been documented [24]. A transperitoneal laparoscopic/robotic approach can be performed extravesically (with dissection of the diverticulum, resection of its neck and suture of the defect) or through a widely opened bladder [10]. The approach through an opened bladder to the diverticulum neck is probably easier, but is hampered by the fact that the bladder defect must be closed subsequently; this is currently the domain of robotic surgery [10]. No studies comparing the above-mentioned techniques are available to serve as evidence-based medicine. We have chosen laparoscopy (a robotic system is not available at our institutions), a transperitoneal approach (the transvesical approach appears too complicated to us) and extravesical - without need of suturing an additional defect of the dissected bladder. In the transperitoneal extravesical approach, applied in our series, the crucial event is to identify the diverticulum and its neck. Some techniques (not applied in our cohort) can help to find diverticulum in an extravesical approach: balloon of catheter in the diverticulum $[1,6$, $9,25]$, illumination by cystoscope [26] and instillation of methylene blue [22].

Of equal importance as the choice of approach to the diverticulum is the method of how to treat the co-existing $\mathrm{BPH}$. A one-step procedure is preferred. There are many treatment options for BPH. Transurethral resection of the prostate has been the gold standard for many years. Iselin et al. [27] combined TURP and LD in 2 cases. They resected only $8 \mathrm{~g}$ of prostatic tissue in both cases. The duration of the diverticulectomy was 4 and $5 \mathrm{~h}$, respectively. Porpiglia et al. [25] combined TURP and LD successfully in 12 cases with a mean operative time of $239 \mathrm{~min}$. Altunrende et al. [28] combined TURP and robotic diverticulectomy; the authors even combined robotic diverticulectomy with robotic simple prostatectomy. Shan et al. combined LD with holmium laser enucleation of the prostate in three cases [29]. The concept of our tech- 
nique was introduced by Kural et al. in 2009 [1] - PVP and mini-invasive (in their case robotic) diverticulectomy. We prefer this combination because we consider PVP to have lower risk of postoperative bleeding than TURP. The concept of using PVP is supported by the recently published GOLIATH study. It showed that PVP performed using the third generation of machine (180-W XPS GreenLight Laser Vaporisation) produced equal results to those of TURP [30].

\section{Conclusions}

Pseudodiverticulum of the urinary bladder (with or without subvesical obstruction) is a relatively rare disease. Due to its rarity, it will be difficult to perform a clinical trial of higher rank than a case report series. A further problem in gaining high quality studies for evidence-based medicine data is the nonexistence of a gold standard. We present a limited study on our preferred treatment method, which is a single-session, two-step procedure using PVP (GreenLight Laser XPS, MoXy fibre) and laparoscopic (transperitoneal extravesical) diverticulectomy. We consider this to be the preferred method for the treatment of subvesical obstruction due to BPH associated with bladder diverticulum. There were no peri- or postoperative complications in our series.

\section{Acknowledgments}

The work was supported by the Czech government research project MSM 0021620819 and by the project Ministry of Health, Czech Republic for conceptual development of research organization 00669806 - Faculty Hospital in Pilsen, Czech Republic.

\section{Conflict of interest}

The authors declare no conflict of interest.

\section{References}

1. Kural AR, Atug F, Akpinar H, Tufek I. Robot-assisted laparoscopic bladder diverticulectomy combined with photoselective vaporization of prostate: a case report and review of literature. J Endourol 2009; 23: 1281-5.

2. Das S. Laparoscopic removal of bladder diverticulum. J Urol 1992; 148: 1837-9.

3. Parra RO, Jones JP, Andrus CH, Hagood PG. Laparoscopic diverticulectomy: preliminary report of a new approach for the treatment of bladder diverticulum. J Urol 1992; 148: 869-71.

4. Anderson KR, Clayman RV. Laparoscopic lower urinary tract reconstruction. World J Urol 2000; 18: 349-54.
5. Faramarzi-Roques R, Calvet C, Gateau T, Ballanger PH. Surgical treatment of bladder diverticula: laparoscopic approach. J Endourol 2004; 18: 69-72.

6. Flasko T, Toth G, Benyo M, et al. A new technical approach for extraperitoneal laparoscopic bladder diverticulectomy. J Laparoendosc Adv Surg Tech A 2007; 17: 659-61.

7. Jarrett TW, Pardalidis NP, Sweetser P, et al. Laparoscopic transperitoneal bladder diverticulectomy: surgical technique. J Laparoendosc Surg 1995; 5: 105-11.

8. Nadler RB, Pearle MS, McDougall EM, Clayman RV. Laparoscopic extraperitoneal bladder diverticulectomy: initial experience. Urology 1995; 45: 524-7.

9. Myer EG, Wagner JR. Robotic assisted laparoscopic bladder diverticulectomy. J Urol 2007; 178: 2406-10.

10. Thuroff JW, Roos FC, Thomas C, et al. Surgery illustrated-surgical atlas: robot-assisted laparoscopic bladder diverticulectomy. BJU Int 2012; 110: 1820-36.

11. Abreu AL, Chopra S, Dharmaraja A, et al. Robot-assisted bladder diverticulectomy. J Endourol 2014; 28: 1159-64.

12. Christman MS, Casale P. Robot-assisted bladder diverticulectomy in the pediatric population. J Endourol 2012; 26: 1296-300.

13. Dindo D, Demartines N, Clavien PA. Classification of surgical complications: a new proposal with evaluation in a cohort of 6336 patients and results of a survey. Ann Surg 2004; 240: 205-13.

14. Thiel DD, Young PR, Wehle MJ, et al. Robotic-assisted bladder diverticulectomy: tips and tricks. Urology 2011; 77: 1238-42.

15. Thwaini A, McLeod A, Nambirajan T. Laparoscopic bladder diverticulectomy. J Laparoendosc Adv Surg Tech A 2008; 18: 849-51.

16. Dobruch J, Piotrowicz S, Skrzypczyk M, et al. Clinical value of extended pelvic lymph node dissection in patients subjected to radical prostatectomy. Videosurgery Miniinv 2014; 9: 64-70.

17. Golabek T, Jaskulski J, Jarecki P, et al. Laparoscopic radical prostatectomy with bladder neck preservation: positive surgical margin and urinary continence status. Videosurgery Miniinv 2014; 9: 362-70.

18. Roslan M, Markuszewski M, Klacz J, Krajka K. Suprapubic transvesical laparoendoscopic single-port bladder diverticulectomy: points of technique with medium-term surgical outcomes. J Endourol 2013; 27: 688-92.

19. Roslan M, Markuszewski M, Kłącz J, et al. Laparoscopic nephroureterectomy with transvesical single-port distal ureter and bladder cuff dissection: points of technique and initial surgical outcomes with five patients. Videosurgery Miniinv 2014; 9 : 267-72.

20. Pansadoro V, Pansadoro A, Emiliozzi P. Laparoscopic transvesical diverticulectomy. BJU Int 2009; 103: 412-24.

21. Eyraud R, Laydner H, Autorino R, et al. Robot-assisted laparoscopic bladder diverticulectomy. Curr Urol Rep 2013; 14: 46-51.

22. Moore CR, Shirodkar SP, Avallone MA, et al. Intravesical methylene blue facilitates precise identification of the diverticular neck during robot-assisted laparoscopic bladder diverticulectomy. J Laparoendosc Adv Surg Techn A 2012; 22: 492-5.

23. Moreno Sierra J, Galante-Romo I, Ortiz-Oshiro E, et al. Bladder diverticulum robotic surgery: systematic review of case reports. Urol Int 2010; 85: 381-5.

24. Stolzenburg JU, Do M, Kallidonis P, et al. Laparoendoscopic single-site bladder diverticulectomy: technique and initial experience. J Endourol 2011; 25: 85-90. 
25. Porpiglia F, Tarabuzzi R, Cossu M, et al. Is laparoscopic bladder diverticulectomy after transurethral resection of the prostate safe and effective? Comparison with open surgery. J Endourol 2004; 18: 73-6.

26. Reboucas RB, Monteiro RC, de Souza TN, et al. Laparoscopic bladder diverticulectomy assisted by cystoscopic transillumination. Int Braz J Urol 2014; 40: 281-2.

27. Iselin CE, Winfield HN, Rohner S, Graber P. Sequential laparoscopic bladder diverticulectomy and transurethral resection of the prostate. J Endourol 1996; 10: 545-9.

28. Altunrende F, Autorino R, Patel NS, et al. Robotic bladder diverticulectomy: technique and surgical outcomes. Int J Urol 2011; 18; 265-71.

29. Shah HN, Shah RH, Hegde SS, et al. Sequential holmium laser enucleation of the prostate and laparoscopic extraperitonea bladder diverticulectomy: initial experience and review of literature. J Endourol 2006; 20: 346-50

30. Bachmann A, Tubaro A, Barber N, et al. 180-W XPS GreenLight Laser Vaporisation versus transurethral resection of the prostate for the treatment of benign prostatic obstruction: 6-month safety and efficacy results of a European Multicentre Randomised Trial-The GOLIATH Study. Eur Urol 2014; 65: 931-42.

Received: 31.07.2014, accepted: 21.01.2015. 